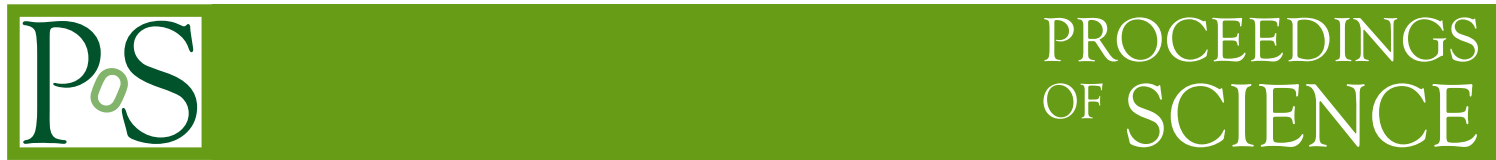

\title{
Bianchi Identities and Degeneracy of Chromomagnetic Fields in SU(2) Gluodynamics
}

\author{
F.V. Gubarev*, S.M. Morozov, \\ ITEP, B.Cheremushkinskaya 25, Moscow, 117218, Russia, \\ E-mail: gubareveitep.ru, smoroz@itep.ru
}

\begin{abstract}
We investigate the non-Abelian Bianchi identities in pure SU(2) lattice Yang-Mills theory in three and four dimensions. The non-Abelian Stokes theorem proposed recently allows to formulate the Bianchi identities in terms of local physical fluxes. Then the violation of Bianchi identities becomes a well defined concept ultimately related to chromomagnetic fields degeneracy points. We present numerical evidences that in $\mathrm{D}=4$ the suppression of the Bianchi identities violation destroys confinement while the removal of the degeneracy points drives the theory to the topologically non-trivial sector.
\end{abstract}

XXIIIrd International Symposium on Lattice Field Theory

25-30 July 2005

Trinity College, Dublin, Ireland

\footnotetext{
${ }^{*}$ Speaker.
} 


\section{Bianchi Identities and Chromomagnetic Fields Degeneracy Points on the Lattice}

The possibility of Bianchi identities violation had always been considered as probable cause of confi nement (see, e.g., Ref. [1] for recent discussion). However, detailed treatment of the problem in non-Abelian case was missing until recently [2] and here we briefly review the main points of this paper. Formally, the Bianchi identities in three and four dimensions

$$
D_{\mu} \tilde{F}_{\mu \nu}=0, \tilde{F}_{\mu \nu}^{a}=\frac{1}{2} \varepsilon_{\mu \nu \lambda \rho} F_{\lambda \rho}^{a}(4 D), \quad D_{i} B_{i}=0, B_{i}^{a}=1 / 2 \varepsilon_{i j k} F_{j k}^{a}(3 D)
$$

look similar, but in fact there is a crucial difference: for given non-Abelian fi eld strength Eq. (1.1) allows to express the gauge potentials as local single-valued functions of $F_{\mu \nu}$, while in three dimensions it does not constraint $A_{\mu}$ at all. The inversion $A(F)$ is possible away from the points chromomagnetic fi elds degeneracy [3], where $\operatorname{det} T=0, T_{\mu \nu}^{a b}=\varepsilon^{a b c} \tilde{F}_{\mu \nu}^{c}$. Note that

$$
\operatorname{det} T \propto \operatorname{det} K, \quad K_{\mu v}=K_{v \mu}=\frac{1}{3} \varepsilon^{a b c} \tilde{F}_{\mu \rho}^{a} F_{\rho \lambda}^{b} \tilde{F}_{\lambda \nu}^{c}
$$

and each element of $K_{\mu \nu}$ is gauge invariant determinant constructed from chromoelectric $E_{i}^{a}$ and chromomagnetic $B_{i}^{a}$ fi elds. It is amusing that our investigation of the Bianchi identities (1.1) and possibility of their violation naturally leads to the same determinants (1.2). Moreover, the degeneracy points turn out to be closely related to the gauge fi elds topology.

We start from the observation [4] that the Bianchi identities (1.1) are equivalent to the requirement that the non-Abelian Stokes theorem (NAST) being applied to closed infi nitesimal surface $S_{0}$ always gives unity on the r.h.s. (surface independence)

$$
\mathrm{P}_{S} \exp \frac{i}{2} \int_{S_{0}, \delta S_{0}=0} \mathscr{F}_{\mu \nu} d^{2} \sigma^{\mu v}=1=e^{i \vec{\sigma} \vec{n} \cdot 2 \pi q}, \quad \vec{n}^{2}=1, \quad q \in Z,
$$

where for defi niteness we used the operator formulation [5] of NAST and r.h.s. was represented in most general form. The color direction $\vec{n}$ is gauge variant and is of no concern. However, the possibility to give unambiguous gauge invariant meaning to the integer $q$ ("magnetic charge") is important since if it is non vanishing then the continuum Bianchi identities are violated somewhere inside $S_{0}$. Note that the term "magnetic charge" here has nothing to do with its usual meaning, in particular, its conservation is neither assumed nor implied (see also below). Since Eq. (1.3) is the defi nition of the magnetic charge the only way to make sense of $q$ is to express the non-Abelian Stokes theorem in terms of gauge invariant physical fluxes.

It is crucial to choose the appropriate parametrization $[2,6]$ for the Wilson loops

$$
\begin{array}{r}
W(C, t)=\operatorname{Pexp} i \int_{t}^{T+t} A(\tau) d \tau=\exp \{i \vec{\sigma} \vec{n}(C, t) \cdot \Phi(C)\}, \\
\vec{n}^{2}(C, t)=1, \quad W(C)=\frac{1}{2} \operatorname{Tr} W(C, t)=\cos \Phi(C),
\end{array}
$$

where $C=\{x(t), 0 \leq t \leq T, x(0)=x(T)\}$ is some closed contour and it is understood that $W(C) \neq$ \pm 1 generically. The flux magnitude $\Phi(C) \in[0 ; \pi]$ is gauge invariant, while the instantaneous flux color direction $\vec{n}(C, t)$ explicitly depends on $t$ and is in the adjoint representation. Note that the flux magnitude is insensitive to the change of contour orientation while the flux direction changes 
sign. Consider now another contour $C^{\prime}$ which touches (or intersects) $C$ at point $x(t)=x^{\prime}\left(t^{\prime}\right)$. Evidently, the relative orientation (angle in between) of $\vec{n}(C, t)$ and $\vec{n}\left(C^{\prime}, t^{\prime}\right)$ remains intact under the gauge transformations. Moreover, the construction could be iterated: for $N$ contours $C_{i}, i=1, \ldots, N$ intersecting at one point the relative orientation of instantaneous fluxes $\vec{n}^{(i)}$ at that point is gauge invariant. In particular, the oriented area ${ }^{1} \Omega_{N}\left(\vec{n}^{(1)}, \ldots, \vec{n}^{(N)}\right)$ of spherical N-vertex polygon is well defi ned.

The next point concerns the relation between the physical fi eld strength and the corresponding infi nitesimal Wilson loop. The basic and well known fact is that the lattice area element $d x^{\mu} d x^{\nu}$ is unoriented $d x^{\mu} d x^{\nu}=d x^{v} d x^{\mu}$ contrary to the usual continuum relation $\delta \sigma^{\mu \nu}=d x^{\mu} \wedge d x^{v}=$ $-d x^{v} \wedge d x^{\mu}$. Therefore in order to defi ne the lattice counterpart of the fi eld strength a canonical orientation of all elementary plaquettes should be fi xed fi rst. Moreover, the canonical ordering is well known, the conventional agreement is to consider $\delta \sigma^{\mu v}$ with $\mu<v$ only. From now on we always assume that the infi nitesimal fluxes are constructed with canonically oriented plaquettes.

Once the canonical orientation is fi xed, the NAST presented and discussed in details in Ref. [6] becomes completely unambiguous. It relates the flux piercing the large contour $C$ with magnitudes and relative orientations of elementary fluxes on (arbitrary) surface bounded by $C$. The requirement of surface independence (1.3) considered for elementary lattice 3-cube $c$ becomes then

$$
\sum_{p \in \delta c} I(p) \Phi(p)+\sum_{x \in \delta c} \Omega_{3}\left(\vec{n}_{x}^{(1)}, \vec{n}_{x}^{(2)}, \vec{n}_{x}^{(3)}\right)=2 \pi q(c),
$$

where $\Phi(p)$ is the plaquette flux, $1 / 2 \operatorname{Tr} W(p)=\cos \Phi(p), \Omega_{3}$ was discussed above and accounts for the difference in color orientations of three fluxes emanating from 3-cube and having one common point $x \in \delta c$. The factors $I(p)= \pm 1$ are the usual incidence numbers: $I(p)=1$ if vertices of $p$ are followed in the canonical order and $I(p)=-1$ otherwise. Eq. (1.5) is the defi nition of the magnetic charge associated with 3-cube and indeed expresses the essence of non-Abelian Bianchi identities. In particular, the continuum Bianchi identities (1.1) are violated inside $c$ if $q(c) \neq 0$.

It is important that the non-Abelian Bianchi identities (1.1) became completely Abelian-like (1.5) in our approach. Moreover, it is possible to construct a specific but unique cell complex in which every term on 1.h.s. of Eq. (1.5) is associated with particular 2-dimensional cell. Then Eq. (1.5) is the usual coboundary operation $d: \mathbb{C}^{2} \rightarrow \mathbb{C}^{3}$ acting on particular 2-cochains. One could say that the non-Abelian nature of (1.1) had been traded for much more complicated geometry underlying (1.5), but which allows purely formal investigation. In particular, it is clear that the 2and 3-skeletons of the cell complex are not exhausted by the original plaquettes and 3-cubes (see Ref. [2] for details). Then the study of $d: \mathbb{C}^{2} \rightarrow \mathbb{C}^{3}$ in its generality leads to the consideration of the "magnetic charges" assigned to all the 3-cells of the complex. It is true that some of these "new" 3-cells are trivial and the corresponding magnetic charge is identically zero. However, there are a non-trivial 3-cells as well which are distinct from the original lattice 3-cubes. One could show that the corresponding charges $\tilde{q}$ signal the zeros of the determinants entering (1.2). Formally the charges $\tilde{q}$ are coming from the $\Omega$ terms in (1.5) and indeed depend only upon flux color directions, not their magnitudes. Note that the union of all magnetic charges $q, \tilde{q}$ is indeed conserved, but the conservation takes place not in the original hypercubical geometry.

\footnotetext{
${ }^{1}$ The total area of unit two-dimensional sphere is taken to be $2 \pi$.
} 

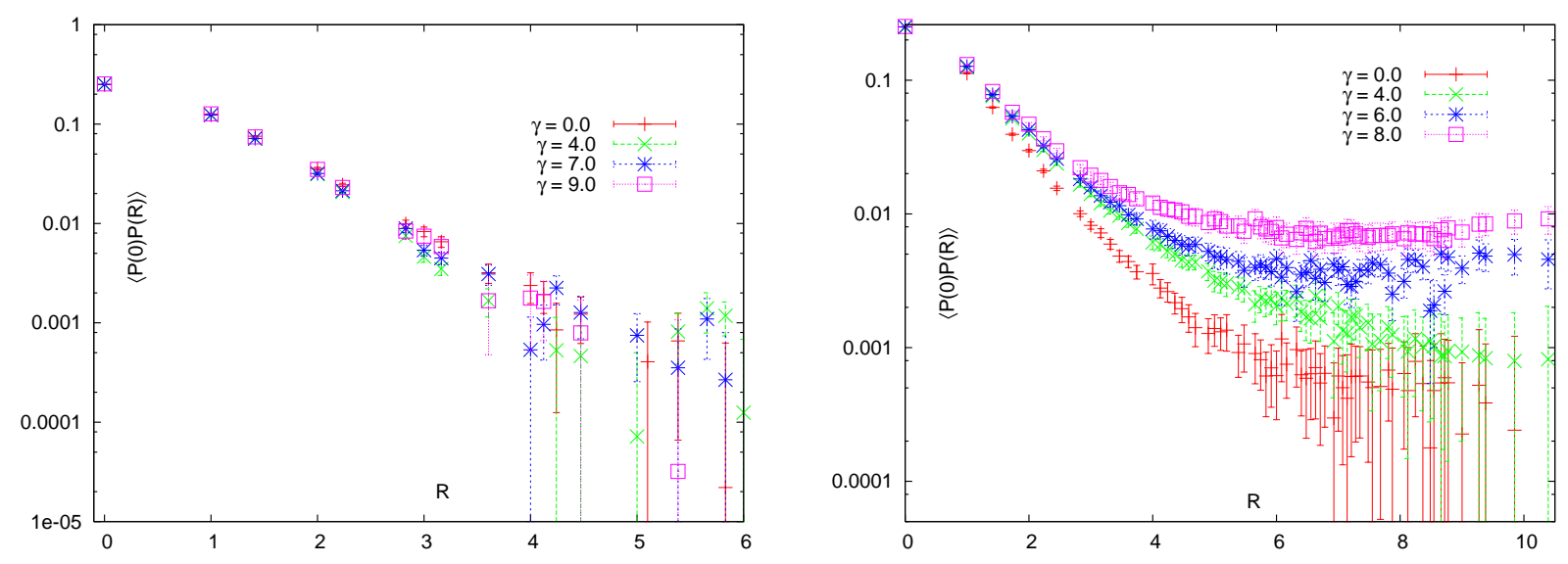

Figure 1: The correlation function of Polyakov lines at $\tilde{\gamma}=0$ and various $\gamma$ in three (left) and four (right) dimensions.

To summarize, the attempt to consider gauge invariant content of the Bianchi identities (1.1) forces us to introduce two types of the "magnetic charges" q, q. First one, Eq. (1.5), is responsible for the Bianchi identities violation and is assigned to lattice 3-cubes. Second one, being assigned to lattice sites, is directly related to the degeneracy points, where a particular determinants constructed from $E_{i}^{a}, B_{i}^{a}$ vanish.

\section{Numerical Experiments}

Simple weak coupling analyzes show that in perturbation theory the $q, \tilde{q}$ charges form tightly bounded dipole pairs, but cannot annihilate because at any fi nite lattice spacing they by defi nition are fi nitely separated. This invalidates the consideration of the corresponding densities since they both would diverge in the continuum limit. On the other hand, the defi nition of $q$ and $\tilde{q}$ charges is local and gauge invariant. Thus we could modify the Wilson action to include additional terms

$$
S=-\beta \sum_{p} \frac{1}{2} \operatorname{Tr} U_{p}+\gamma \sum_{c}|q(c)|+\tilde{\gamma} \sum_{s}|\tilde{q}(s)|,
$$

which suppress the charges $q, \tilde{q}$ and allow to study their physical relevance. The particular limit $\gamma \rightarrow$ $\infty$ is of special interest since it corresponds to the theory with nowhere violated Bianchi identities. As far as the $\tilde{\gamma}$ coupling is concerned the limit $\tilde{\gamma} \rightarrow \infty$ might not correspond to sensible theory. For instance, the nowhere vanishing $\operatorname{det} B$ implies that it is of the same sign everywhere, which contradicts the perturbative expectations and probably violates $C P$ symmetry.

The model (2.1) was studied in $D=3,4$ along the lines $\tilde{\gamma}=0, \gamma=0$ at fi xed value of the gauge coupling $\beta$. The simulations were performed on $20^{3}$ and $12^{4}$ lattices at $\beta=6.0$ and $\beta=2.4$ correspondingly. We implemented the Metropolis algorithm which is the only one available at non-zero $\gamma, \tilde{\gamma}$. The procedure turns out to be very time consuming. Because of this the volumes were not so large and only the limited set of $(\gamma, \tilde{\gamma})$ points were considered. At each $(\gamma, \tilde{\gamma})$ values we generated about one hundred statistically independent gauge samples separated by $\sim 10^{3}$ Monte Carlo sweeps. The observable of primary importance is the correlator of the Polyakov lines 



Figure 2: Left: topological susceptibility in $\mathrm{D}=4$ at $\tilde{\gamma}=0$ and various $\gamma$. Right: Monte Carlo history of global topological charge at $\gamma=0$ and two $\tilde{\gamma}$ values.

$\langle P(0) P(R)\rangle$ for which the standard spatial smearing [7] and hypercubic blocking [8] for temporal links were used. In $\mathrm{D}=4$ we also monitored the topological charge $Q$ and the topological susceptibility $\chi=\left\langle Q^{2}\right\rangle / V$ measured with overlap Dirac operator [9] (see, e.g. Ref [10] for review and further references).

Consider the response of the heavy quark potential on rising $\gamma$ coupling. In three dimensions (Fig. 1, left) the correlator $\langle P(0) P(R)\rangle$ shows almost no sign of $\gamma$ coupling dependence, in particular, the asymptotic string tension at large $\gamma$ is equal to its value in the pure Yang-Mills limit. However, the situation changes drastically in $\mathrm{D}=4$. It is apparent (Fig. 1, right) that the correlation function $\langle P(0) P(R)\rangle$ tends to non-zero positive value at large separations

$$
\lim _{R \rightarrow \infty}\langle P(0) P(R)\rangle_{\gamma \gtrsim 1}=\text { const }>0,
$$

when $\gamma$ coupling becomes of order few units. On the other hand, other measured observables do not show strong dependence on $\gamma$ coupling. In particular, the topological charge $Q$ stays at zero in average albeit with slightly narrower distribution. As is clear from Fig. 2 (left) the topological susceptibility drops at $\gamma \approx 1$ by approximately $25 \%$ and then stays almost constant, $\lim _{\gamma \rightarrow \infty} \chi^{1 / 4}(\gamma)=163(8) \mathrm{MeV}$. Therefore the dynamics of YM fi elds in $\mathrm{D}=3$ seems to be almost insensitive to whether or not the Bianchi identities are violated. Contrary to that, in $D=4$ case the suppression of the Bianchi identities violation seems to destroys confi nement while other measured characteristics of the theory remain essentially unchanged. For interpretation and more details see Ref. [2].

As we noted already the suppression of degeneracy points might not be physically meaningful. Indeed, we found that at any $\tilde{\gamma}>0$ the Polyakov lines correlator becomes an oscillating function of the distance signaling the reflection positivity violation. In four dimensional case the natural reason of this is non-zero in average global topological charge. The right panel on Fig. 2 shows the Monte Carlo history of the topological charge on $8^{4}$ lattice at $\beta=2.30, \gamma=0, \tilde{\gamma}=0.1,0.5$ when the starting confi guration was thermalized at $\gamma=\tilde{\gamma}=0$ (the lattice geometry and the $\beta$ coupling were changed for reasons to be explained shortly). In particular, for $0<\tilde{\gamma} \ll 1$ the mean topological 
charge is shifted only slightly being of order few units. However, once the $\tilde{\gamma}$ coupling becomes comparable with unity $Q$ flows away from zero during Monte Carlo updating towards an extremely large and positive values with almost constant and very high rate. In fact, it quickly becomes too large to be technically accessible for us and this was essentially the reason to consider so small lattices here. The volume dependence of $\langle Q\rangle$ could be inferred by noting that the last term in (2.1) responsible for the rapid increase of the topological charge is the bulk quantity. Therefore $\langle Q\rangle$ is expected to be proportional to the volume at fi xed $\tilde{\gamma}$ although we had not investigated this numerically.

To conclude we note that the numerical results confi rming the connection between confi nement and violation of Bianchi identities should be improved in order to be convincing. In particular, we hope to study elsewhere the larger volumes and $\beta$ coupling. Nevertheless, our data clearly indicates the relation between the degeneracy points and the gauge fi elds topology, which is worth to be further investigated.

\section{References}

[1] A. M. Polyakov, “Confining strings” Nucl. Phys. B 486, 23 (1997) [hep-th/9607049].

[2] F. V. Gubarev and S. M. Morozov, “ $\left\langle A_{\text {min }}^{2}\right\rangle$ condensate, Bianchi identities and chromomagnetic fields degeneracy in SU(2) YM theory”, Phys. Rev. D 71, 114514 (2005) [hep-lat/0503023].

[3] S. Deser and C. Teitelboim, "Duality Transformations Of Abelian And Nonabelian Gauge Fields", Phys. Rev. D 13 (1976) 1592; R. Roskies, "On The Uniqueness Of Yang-Mills Potentials”, Phys. Rev. D 15, 1731 (1977); M. B. Halpern, "Field Strength Formulation Of Quantum Chromodynamics", Phys. Rev. D 16, 1798 (1977); M. B. Halpern, "Gauge Invariant Formulation Of The Selfdual Sector”, Phys. Rev. D 16, 3515 (1977).

[4] J. E. Kiskis, “The Bianchi Identity For Nonabelian Lattice Gauge Fields”, Phys. Rev. D 26, 429 (1982).

[5] I. Arefeva, "Nonabelian Stokes Formula", Theor. Math. Phys. 43, 353 (1980) [Teor. Mat. Fiz. 43, 111 (1980)]; N. E. Bralic, "Exact Computation Of Loop Averages In Two-Dimensional Yang-Mills Theory”, Phys. Rev. D 22, 3090 (1980).

[6] F. V. Gubarev, V. I. Zakharov, "The Berry phase and monopoles in non-Abelian gauge theories”, Int. J. Mod. Phys. A 17, 157 (2002) [hep-th/0004012]; F. V. Gubarev, V. I. Zakharov, "Gauge invariant monopoles in SU(2) gluodynamics", hep-lat/0204017; F. V. Gubarev, "On the non-Abelian Stokes theorem for SU(2) gauge fields”, Phys. Rev. D 69, 114502 (2004), [hep-lat/0309023]; "Gauge invariant monopoles in lattice SU(2) gluodynamics", hep-lat/0204018.

[7] M. Albanese et al. [APE Collaboration], "Glueball Masses And String Tension In Lattice QCD”, Phys. Lett. B 192, 163 (1987).

[8] A. Hasenfratz and F. Knechtli, "Flavor symmetry and the static potential with hypercubic blocking", Phys. Rev. D 64, 034504 (2001) [hep-lat/0103029].

[9] H. Neuberger, "Exactly massless quarks on the lattice”, Phys. Lett. B 417, 141 (1998) [hep-lat/9707022]; “More about exactly massless quarks on the lattice”, Phys. Lett. B 427, 353 (1998) [hep-lat/9801031].

[10] L. Giusti, C. Hoelbling, M. Luscher and H. Wittig, "Numerical techniques for lattice QCD in the epsilon-regime”, Comput. Phys. Commun. 153, 31 (2003) [hep-lat/0212012]. 